\title{
Was wenn kein intravenöser Zugang gelingt?
}

Fragestellung: Anhand einer Metaanalyse soll geklärt werden, welches der nicht intravenös applizierbaren Benzodiazepine in der Anfalls- und Statusdurchbrechung wirksam ist, zum einen im direkten Vergleich untereinander, zum anderen im Vergleich zu intravenös appliziertem Diazepam.

Hintergrund: Der prolongierte generalisierte Anfall und der konvulsive Status epilepticus sind Notfälle, die mit hoher Mortalität und bei Überleben der Akutsituation mit hohen Komplikationsraten und Folgeerkrankungen einhergehen können. Relevant für das Outcome ist das rasche Durchbrechen des Status. First-line werden Benzodiazepine verabreicht. Doch welches der verfügbaren Präparate ist sinnvollerweise zu nehmen, wenn kein zuverlässiger intravenöser Zugang gelegt werden kann?

Patienten und Methodik: Diese Frage prüfte ein systematischer Review und eine Metaanalyse randomisierter kontrollierter Studien, in denen mindestens Arya R, Kothari $\mathrm{H}$, Zhang Z et al. Efficacy of nonvenous medications for acute convulsive seizures. A network meta-analysis Neurolog. 2015; 85: 1859-68 ein Behandlungsarm eine nicht intravenöse Medikation erhielt. Ausgewertet wurden Studien, die sublinguales Lorazepam (SL-LOR), intravenöses Lorazepam (IV-
LOR), intranasales Lorazepam (IN-LOR), bukkales Midazolam (BC-MDZ), intranasales Midazolam (IN-MDZ), intramuskuläres Midazolam (IM-MDZ), rektales Diazepam (PR-DZP) und intramuskuläres Paraldehyd (IM-PLD) verwendet hatten.

Ergebnisse: In 16 Studien war insgesamt IM-MDZ von allen nicht intravenös applizierbaren Präparaten am effektivsten zur Anfallsdurchbrechung. Wurde ein 10-Minuten-Fenster betrachtet, schnitt IN-MDZ unter den nicht intravenösen Präparaten ebenfalls sehr gut ab, auch was die anhaltende antikonvulsive Wirkung über $\geq 1$ Stunde nach Anfallsende betraf. Die Dauer bis zur Anfallsdurchbrechung lag bei IM-MDZ bei durchschnittlich 2,14, bei IN-MDZ bei 2,44 und bei BC-MDZ bei 4,3 Minuten. IM-MDZ war im direkten Vergleich zwar schneller als rektales Diazepam wirksam, aber nicht dem intranasalen MDZ oder intravenös applizierten Diazepam überlegen. Auch war der Zugriff auf IM-MDZ schneller als auf IV-LOR (1,2 vs. 4,8 Minuten), was sich auf die Gesamtdauer des Anfalls positiv auswirkte.

Schlussfolgerungen: Intramuskulär sowie intranasal appliziertes Midazolam sind in der Geschwindigkeit und Nachhaltigkeit der Wirkung zur Durchbrechung von prolongierten generalisierten Anfällen inklusive des Status epilepticus im Vergleich zu intravenösem Diazepam eine hochwirksame Alternative.

\section{- Kommentar von Vivien Homberg, Bad Berka}

\section{Welches Benzodiazepin in welcher Situation?}

Gute Fragestellung, da gerade prolongierte tonisch-klonische Anfälle oder generalisierte Status epileptici eine Herausforderung darstellen, einen korrekt liegenden intravenösen Zugang zu bekommen. Auch besteht häufig das Problem, dass geschulte Laien sinnvollerweise etwas tun wollen, bevor der Notarzt eintrifft, womit intravenöse Präparate ausfallen. Hier eignet sich natürlich besonders die nasale und bukkale Applikation. Meiner Ansicht nach sollten rektale Applikationen in der Öffentlichkeit bei dieser Fülle an wirksamen Alternativen definitiv nicht mehr verordnet oder empfohlen werden, denn sie sind weder praktikabel noch würdig für alle Beteiligten.

Noch unverständlicher ist die Verordnungsgewohnheit von PR-DZP, wenn man die Head-to-head-Analysen betrachtet, in denen PR-DZP deutlich schlechter abschneidet als das bukkal, nasal oder intramuskulär applizierte Midazolam. Unter anderem gelingt es mit PR-DZP auch nur in $70 \%$ der Fälle, Anfälle innerhalb von zehn Minuten zu durchbrechen, dies bei zusätzlich fehlendem anhaltendem Effekt über die folgenden Stunden (nur noch zirka 55\% Wirkung nach einer Stunde).

In Gesamtbetrachtung aller verfügbaren nicht intravenösen Medikamente muss auch zum sublingual verabreichbaren Lorazepam eine Einschränkung gemacht werden. So sei explizit darauf hingewiesen, dass dies zwar einfach zu handhaben ist, aber in Notfallsituationen aufgrund seines viel zu späten Wirk- eintritts wenig Sinn macht. Hingegen ist es aufgrund seiner besonders langen Halbwertszeit (bis 16 Stunden) ein ideales Präparat, wenn Patienten zu Anfallsserien neigen. Ebenfalls ein Problem stellt das hier erwähnte und gut wirksame intranasale Midazolam dar, da es auf dem deutschen Markt leider weiterhin nicht zur Verfügung steht. Grundsätzlich möglich ist es, die intravenöse Lösung per Zerstäuber nasal zu applizieren.

Von allen genannten Alternativen ist zusammenfassend für den Laien das bukkale Midazolam, für den Ersthelfer im Rettungsdienst oder der Notaufnahme das intramuskuläre Midazolam, zu präferieren, wenn kein intravenöser Zugang möglich ist. Es sind hocheffektive, dem intravenös applizierten Diazepam kaum oder gar nicht unterlegene Präparate.

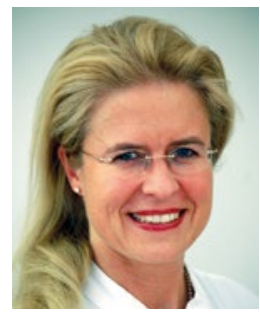

Dr. med. Vivien Homberg, Bad Berka

Chefärztin der Klinik für Neurologie, Zentralklinikum Bad Berka E-Mail: Vivien.Homberg@zentralklinik.de 